\title{
Some Results at Ngomahuru Leprosy Hospital, S. Rhodesia.
}

B. MOISER.

At this voluntary Government hospital patients are photographed upon admission, and at suitable intervals, as it is considered that photographs constitute the best means of recording progress.

The patients have averaged

on 1st January being 515 , with 407 on 31 st December, a difference of 108. 79 new cases were admitted; 6 returned for further treatment; 3 were re-admitted for economic reasons, i.e. they found that their home had disappeared whilst they were in hospital, and they had no friends to go to; 159 were discharged; 32 died; and 5 deserted.

Leprosy is a very mild disease in Southern Rhodesia, the majority of cases being $\mathrm{N}^{1} \mathrm{C}^{1}$ or $\mathrm{N}^{2}$ types. Many abortive cases occur. The purely cutaneous type is non-existent, and I have come to the conclusion that every case begins in this country with neural symptoms.

The number of cases who show little or no improvement, or who retrogress, is very small.

All are natives of Africa, including 2 Dutch, and 1 Coloured.

The routine treatment for natives has been with intramuscular injections of Burroughs Wellcome's Moogrol, in doses of 5 to 10 c.c. over periods of 6 weeks, followed by fortnightly intervals.

The Dutch and Coloured patients have been given Bayer's Preparation No. 4828 A.J. for most of the time, but this has lately been changed to Bayer's " Jantol ", under which one patient, $\mathrm{N}^{1} \mathrm{C}^{1}$, is making remarkable progress after only 10 weeks treatment.

CASE I. Mavonera, (see figs 1 and 2) native roman aged about 40, type $\mathrm{N}^{1} \mathrm{C}^{2}$ Admitted 20.1.34.

Gives a history of only 1 year-probably longer. Nodules on face, both forearms, thighs and legs. Both ulnars and both peroneals thickened and tender.

Anaesthesia. Both uppers. Back of both hands and 5th fingers. Lowers, lower $\frac{1}{3}$ of both peroneals and dorsum of both feet. L.ft. Ear, Plus 1. Only one group seen. Rt. Nose, Negative. Nodule, Plus 5.

28.5.34. Nodules and infiltrations very large. Lft. Ear, Positive. Masses.

1.10.34. Very great improvement. All nodules almost disappeared, leaving only discolouration. 
Anaesthesia, As before; Lft. Ear, Negative.

7.6.35. No skin signs; Lft. Ear, Negative; Lft. Nose, Negative.

9.9.35. Both ulnars still enlarged, but no tenderness; Peroneals not enlarged or tender.

Anaesthesia, Backs of both hands; Lower, Both from mid-thigh down; Rt. Ear and both sides nose, Negative.

17.2.36. No nerve thickening or tenderness.

Anaesthesia, Both Uppers, Back of both hands and all digits.

Lowers, Both peroneal areas from knee; No skin signs whatever; Rt. Ear, Positive Plus 1; Very scanty small groups; Lft. Nose, Negative.

5.10.36. No skin signs and no nerve thickening or tenderness. Anoesthesia, As before; Ear and Nose, Negative.

22.2.37. No signs; Ear and Nose, Negative; Remains in hospital.

Case II. Ndayabaya, (see figs. 3 and 4) native woman aged about 35. Has 7 children all healthy. No mutilations. Admitted 25.2.34. Type $\mathrm{N}^{2} \mathrm{C}^{2}$.

Gives history of few months only. (Histories are notoriously unreliable.)

Skin, Shows large areas of infiltration and nodulation on face, ears, and outer aspects of all limbs, especially the Lft. upper; Hands and feet much swollen; Large perforation nasal septum; Palate thickened and hypopigmented; No ulceration.

Nerves. Uppers, Both ulnars a little thickened and a little tender; Lft., more so; Lowers, Rt. peroneal thickened, and both tender.

Anaesthesia. Uppers, Rt. Ulnar border, back of hand and all digits ; Lft. from shoulder, back of hand and all digits; Lowers, Outer aspect, both lowers from hip down; Ear, Nose and Skin, All plus 4.

4.10.34. Much improved; Nodules on cheeks, neck, Lft. upper arm are smaller and many disappeared.

11.6.35. Remarkable improvement; Nodules and infiltration very largely disappeared from face and upper arms.

Nerves, No nerve thickening or tenderness.

Anaesthesia. Uppers, Rt., Nil ; Lft. Back of hand and all digits.

Lowers, R. Nil; Lft. Lower $\frac{1}{2}$ peroneal area and dorsum; Rt. Ear plus 3; Numerous globi and masses.

20.2.36. Rt. Ear plus 3; Large groups; Bacilli showing fragmentation.

25.2.37. Rt. Ear plus 3 ; Large and small groups; B much dotted; Skin less infiltrated and nodular.

This woman has shown continuous improvement since treatment started, and I have hopes that she will eventually become negative and may even be discharged.

In all smears, a careful note is made of the appearance of the mycobacteriae, for I am of opinion that this is a measure of progress. Advanced cutaneous cases, such as this one, always show at first large closely-packed globi, which stain deeply. As progress takes place, the globi become less dense, and more loosely packed (what I call "groups"), and the bacilli become smaller, and appear dotted, instead of as continuous rods. Finally, it is quite common here to find merely small dots, which may or may not be arranged in a linear fashion. 
Case III. Madhledzo. (See figs. 5 and 6). Tuberculoid variety. Admitted 1.3.35. Native woman aged about 30. Gives a history of 2 months.

Skin, Large raised hyperaemic macule, much thickened, and scaly in parts. Margins very well defined, and markedly raised, and those on the face have the peculiar purple appearance which I have become accustomed to regard as typical of the tuberculoid variety of leprosy.

A good deal of general infiltration of face, especially upper eyelids, lips and nose; Feet much swollen; T.99.

Nerves. Uppers, Slight thickening of both ulnars, and some tenderness; Lowers, Both peroneals markedly thickened and tender.

Anaesthesio, All maculae, and practically both ulnar and peroneal areas; Some nasal ulceration on both sides; Ears, Nose, Maculae, All negative. Impossible to find a bacillus anywhere by usual methods, even in nasal smears.

18.6.35. Remarkable improvement; All puffiness of face, and raised condition of maculae gone; Ears, nose, maculae, negative.

19.3.36. Maculae still plainly visible, only slightly hypopigmented, and edges raised in parts only; No enlargement or tenderness of ulnars; Rt. peroneal a little tender, pain referred to 5 th toe.

Anaethesia, Much less; All smears again negative.

25.2.37. No nerve thickening or tenderness.

Amaesthesio. Uppers, Rt. 1.4 and 5 digits; Lft. Ulnar border from elbow, 4 and 5 digits; Lozers, Rt. Dorsum of foot.

Lft. Lower $\frac{1}{2}$ of leg and dorsum; Ear, nose, maculae, all negative.

These notes have necessarily been condensed from the case book, but they are sufficient to indicate the notable improvement, which is much better shown in the photographs.

This is a typical case of tuberculoid leprosy, as seen here. Such cases are rare here, but they appear to be the most easily amenable to treatment.

The above 3 cases are good examples of results obtained here, but they are not by any means unique. Several others could be quoted, but I have purposely chosen 3 advanced cases, whose changes can be exhibited photographically.

Early cases do well here. They ane all neurals without any striking skin changes, which would be evident in monochrome photographs.

I have now been here for eight years as full-time leprologist, after 20 years in Northern Nigeria, where leprosy was only a part of my duties, but where I nevertheless had a good deal of experience of the disease, with entirely negative results, so that there are evidently one or more factors at work in Southern Rhodesia which produce such gratifying results. This, however, cannot be discussed here.

Whatever these factors may be, it is certain that we have a very fine climate here, and it has been suggested that this large Estate of about 9,000 acres, which comprises the grounds of Ngomahuru, could be put to good use by making it a leprosarium for the British Empire. 

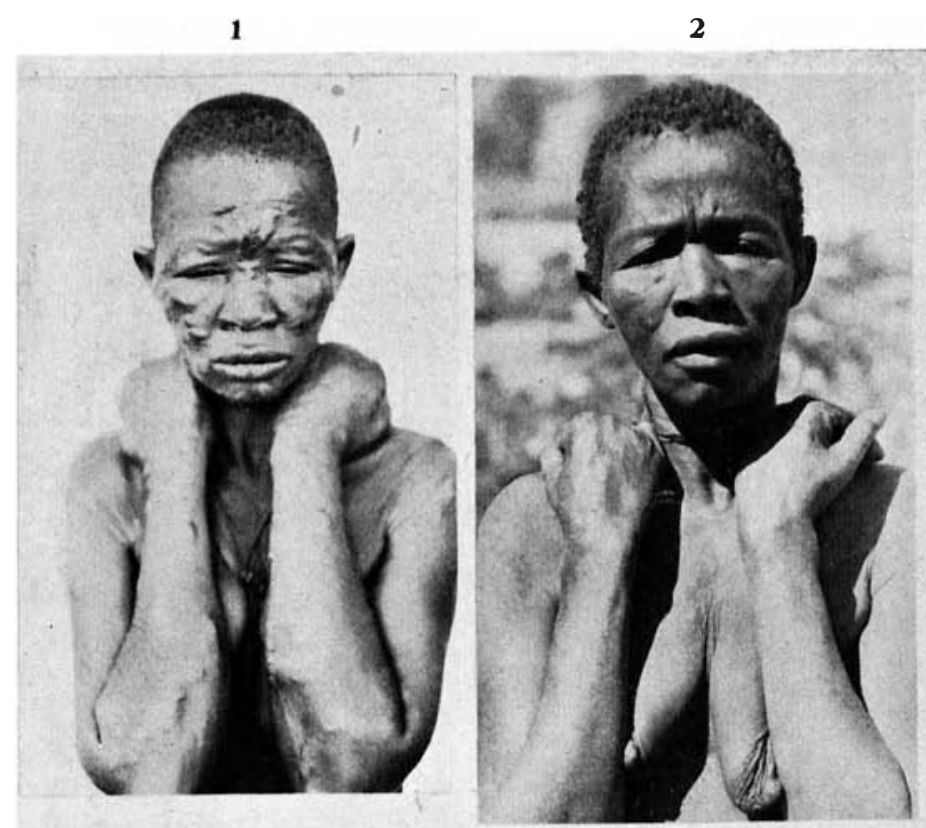

3

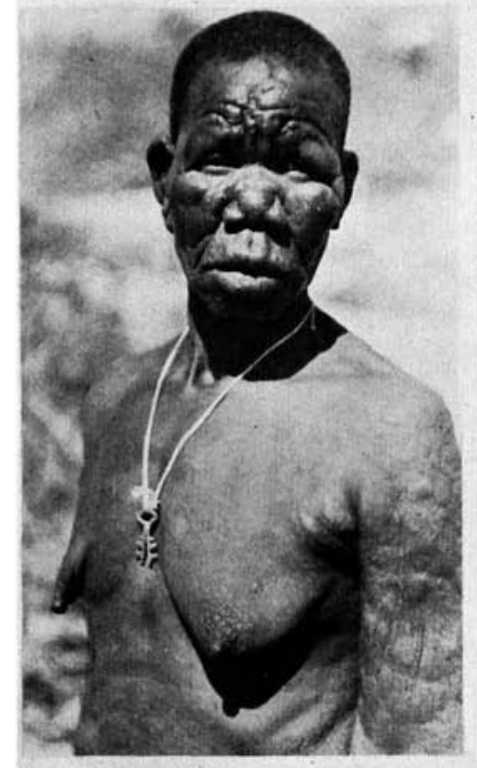

4

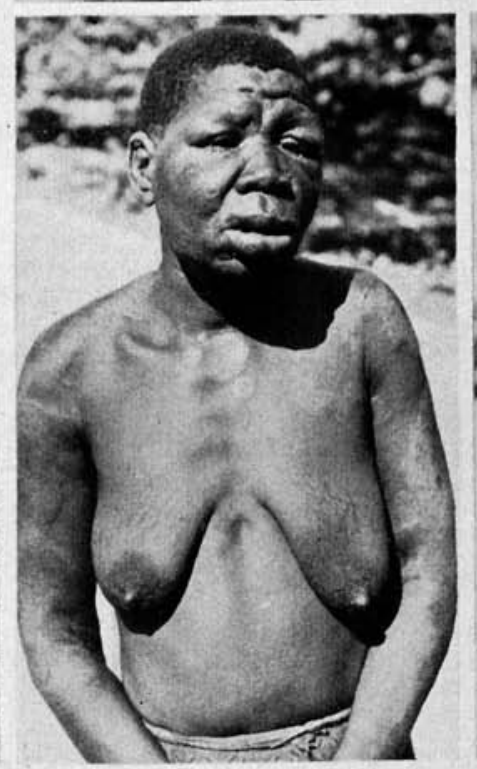

5
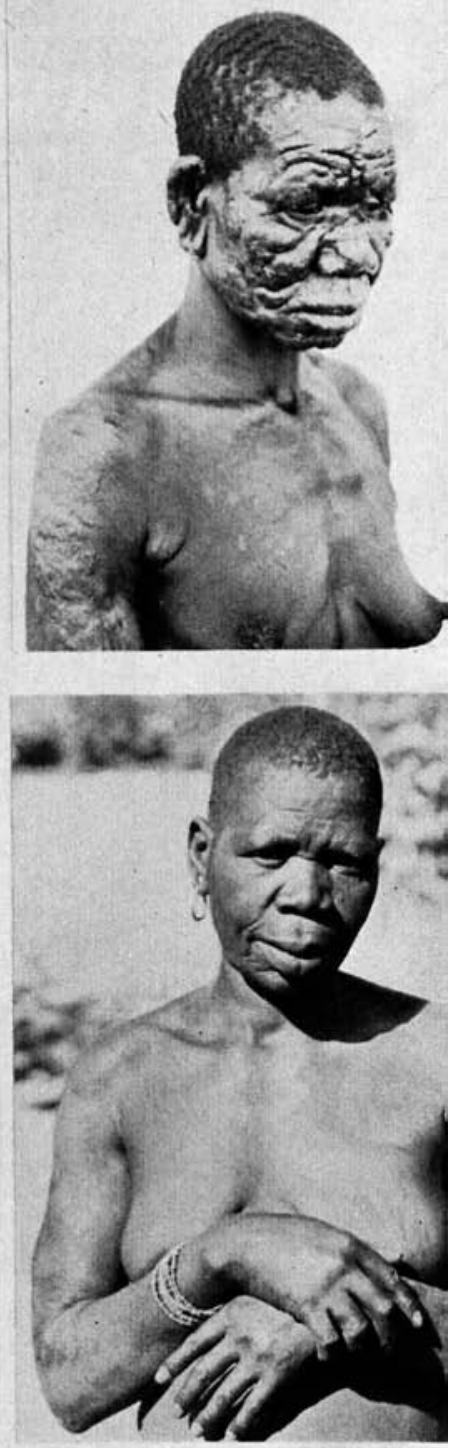

6

Figs. 1 and 2 show effects of treatment in Case 1.

, 3 and $4, \quad, \quad, \quad, \quad$, Case II.

, 5 and $6, \quad, \quad, \quad, \quad$, Case 111. 


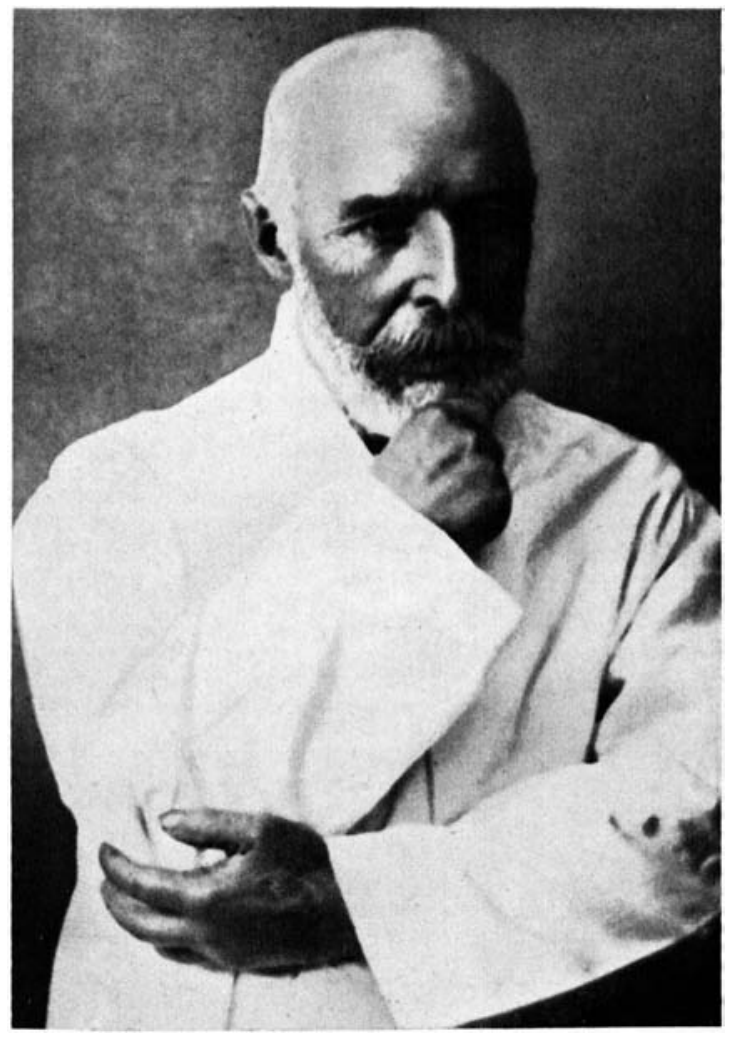

Dr. Armauer hansen 